University of Wollongong

Research Online

Faculty of Engineering and Information

Faculty of Engineering and Information

Sciences - Papers: Part A

Sciences

$1-1-2016$

\title{
Particle size segregation of bulk material in dustiness testers via DEM simulation
}

Sathaphon Wangchai

University of Wollongong, sw741@uowmail.edu.au

David B. Hastie

University of Wollongong, dhastie@uow.edu.au

Peter W. Wypych

University of Wollongong, wypych@uow.edu.au

Follow this and additional works at: https://ro.uow.edu.au/eispapers

Part of the Engineering Commons, and the Science and Technology Studies Commons

Research Online is the open access institutional repository for the University of Wollongong. For further information contact the UOW Library: research-pubs@uow.edu.au 


\title{
Particle size segregation of bulk material in dustiness testers via DEM simulation
}

\author{
Abstract \\ Dustiness testers are used to quantify the level of dust within a bulk material. The generation of dust can \\ occur as a result of many bulk material handling activities, including filling or discharge from storage bins, \\ during free-fall, material impact on conveyor transfers, or impact with other materials. Dust generation \\ can have a serious impact to the environment as well as workers and nearby communities. This paper \\ presents the findings of a discrete element modeling (DEM) study of the flow behavior of a range of \\ binary granular mixes tested in the European Standard and the Australian Standard dustiness testers. Due \\ to current computing limitations, a nondusty material has been chosen for these simulations to determine \\ the underlying characteristics of particle migration within the rotating drums. A range of simulations have \\ been performed using different starting positions of the simulated test product within the rotating drums \\ to determine if this has any overall effect on the particle interactions. Additionally, simulated binary \\ mixtures containing varied size ratios of the same product have been used in an attempt to uncover \\ possible trends, especially in terms of axial and radial segregation.

\section{Keywords} \\ simulation, dustiness, bulk, via, testers, segregation, dem, size, material, particle \\ Disciplines \\ Engineering | Science and Technology Studies

\section{Publication Details} \\ Wangchai, S., Hastie, D. B. \& Wypych, P. W. (2016). Particle size segregation of bulk material in dustiness \\ testers via DEM simulation. Particulate Science and Technology: an international journal, Online First 1-9.
}




\title{
Particle Size Segregation of Bulk Material in Dustiness Testers via DEM Simulation
}

\author{
S. Wangchai, D.B. Hastie ${ }^{\#}$ and P.W. Wypych \\ School of Mechanical, Materials and Mechatronic Engineering, University of \\ Wollongong, Northfields Avenue, Wollongong NSW 2522, Australia \\ ${ }^{\#}$ Corresponding Author: dhastie@uow.edu.au
}

\begin{abstract}
Dustiness testers are used to quantify the level of dust within a bulk material. The generation of dust can occur as a result of many bulk materials handling activities, including; filling or discharge from storage bins, during free-fall, material impact on conveyor transfers or impact with other materials. Dust generation can have a serious impact to the environment as well as workers and nearby communities.

This paper presents the findings of a discrete element modelling study of the flow behaviour of a range of binary granular mixes tested in the European Standard and the Australian Standard dustiness testers. Due to current computing limitations, a non-dusty material has been chosen for these simulations to determine the underlying characteristics of particle migration within the rotating drums. A range of simulations have been performed using different starting positions of the simulated test product within the rotating drums to determine if this has any overall effect on the particle interactions. Additionally, simulated binary mixtures containing varied size ratios of the same product have been used in an attempt to uncover possible trends, especially in terms of axial and radial segregation.
\end{abstract}

Keywords: Discrete element method (DEM), dustiness tester, particle flow, axial segregation, radial segregation

\section{Introduction}

Dust generation is a common occurrence in many bulk materials applications. The emission of dust during handling is dependent on factors such as; the type of material, size distribution of generated particles and properties of the material (Wypych and Cooper, 1995). Some of the issues associated with dust generation or emissions include; health effects on workers, environmental pollution and the wear of machinery (Hjemsted and Schneider, 1996; Hamelmann and Schmidt, 2005; Wypych et al., 2005). One method of quantifying the dustiness of a material is by performing dustiness testing (e.g. rotating drum methods) (Hjemsted and Schneider, 1996; Breum, 1999).

This paper reports on an investigation into the granular material flow in the European Standard (ES) dustiness tester (EN 15051, 2006) and the Australian Standard (AS) dustiness tester (AS 4156.6, 2000) via discrete element modelling (DEM) simulation. It should be noted that the AS tester was originally designed for the determination of the dustiness of coal, however, there is no reason that this tester cannot be used to determine the dustiness of other materials. Dustiness testers also require the use of a vacuum to entrain air through the drums as they rotate, collecting fine dust particles in the process. For the investigation presented here, the air effects are not being considered due to the 
added complexities of incorporating couple computational fluid dynamics. This will be addressed in the future, instead, only the particle motion is being studied at present.

The geometry of the dustiness testers is similar to that of horizontal cylindrical drum mixers, which have been investigated extensively, as presented in the literature. Wightman and Muzzio (1998a) studied the mixing of powders with uniform size in a cylinder and the segregation of particles of unequal sizes in a cylindrical drum (Wightman and Muzzio, 1998b). Other investigations in drum mixers include; the mixing of non-ideal powders (Santomaso et al., 2004), mixing and segregation of granular material (Muzzio et al., 1997; Ottino and Khakhar, 2000; Sudah et al., 2005; Arratia et al., 2006) and the effect of cohesion in mixing and segregation of binary mixtures of uniform and non-uniform size in rotating drums (Dury and Ristow, 1999; Chaudhuri et al., 2006).

Segregation occurs in two main forms in horizontal drum mixers; axial and radial. Elperin (1999) found that axial segregation was more easily achieved when a drum was filled more than $50 \%$ and an added influence was the differences in angle of repose of the small and large particles. Also, when the drum is rotated at a low speed, the particles have low energy and as a result segregation is minimal (Cui et al., 2014).

Axial segregation in horizontal drum mixers subjected to a variety of rotational speeds has led to a range of segregation patterns forming (Arntz et al., 2013) and the added investigation of particle size ratio and fill level has also been evaluated by Kuo et al. (2005), again showing variations in the segregation banding occurring axially. Discrete element modelling has also been used to investigate the formation of axial segregation in horizontal drum mixers (Chen et al., 2011; Cui et al., 2014) and the same bands seen experimentally have been reproduced in the simulations.

Radial segregation also occurs in rotating drum mixers and is a mechanism observed when mixing particles of different sizes. A central core of the fine particle fraction is observed to form, surrounded by the larger particles (Clement et al., 1995). Cantelaube and Bideau (1995) noted that in less than 1 revolution at a low speed of $1.3 \mathrm{rpm}$, a binary mixture of granular disks had segregated, with the smaller particles forming a central cluster. Pollard and Henein (1989) commented that other researchers had found that radial segregation is complete in up to 10 revolutions.

Mixers generally have higher fill volumes than dustiness testers and Chen (2009) found that in investigating $20 \%$ to $60 \%$ fill levels as well as rotational speeds ranging from $2 \mathrm{rpm}$ to $30 \mathrm{rpm}$ that different segregation patterns were possible. At low fill levels a small-large-small axial segregation formed, whereas for higher fill levels large-small-large axial segregation formed. Alexander (2004) found that for cylinders filled to $50 \%$ capacity with a binary granular mix, axial and radial segregation occurred for the majority of testing.

These axial and radial segregation mechanisms will be investigated in the ES and AS dustiness testers through DEM simulation. DEM is now commonly used to simulate a wide range of bulk materials applications (Cleary, 2001; Cleary et al., 2003; Goda and Ebert, 2005; Cleary and Sinnott, 2008; Liu et al., 2008; Ketterhagen et al., 2009; Hastie and Wypych, 2010; Hilton and Cleary, 2011). The principle of DEM is to track, in a time stepping simulation, the position and rotation of each particle element in a system and then to calculate the interactions between the elements 
themselves and also between the elements and their environment. To ensure representative simulations of real-world problems can accurately be predicted, calibrated material models must be used (Grima et al., 2011; Hastie, 2013).

\section{Experimental Dustiness Testing}

Although this paper focusses on simulation, it is prudent to include a section describing the physical attributes and operation of the experimental equipment from which the simulation work is derived. This section presents some representative results of previously completed experimental investigations (Wangchai et al., 2015).

\subsection{Test Apparatus}

The European Standard (ES) and Australian Standard (AS) dustiness testers are shown in Figure 1. For the DEM analyses described later in this paper, the drums have been divided into five equal volumetric sections (virtual). The ES tester operates at a rotational speed of $4 \mathrm{rpm}$ while the AS tester operates at $29 \mathrm{rpm}$. This difference in rotation speed is in part due to the different applications of the testers and also the quantity of material used. The vacuum mechanism has been disabled in both testers so that a study of the behaviour of the particle motion in both rotating drums can be completed. This simplifies the process of producing discrete element modelling (DEM) simulations without the added need to incorporate computational fluid dynamics (CFD) and coupled simulations with DEM, which will be presented in future research.
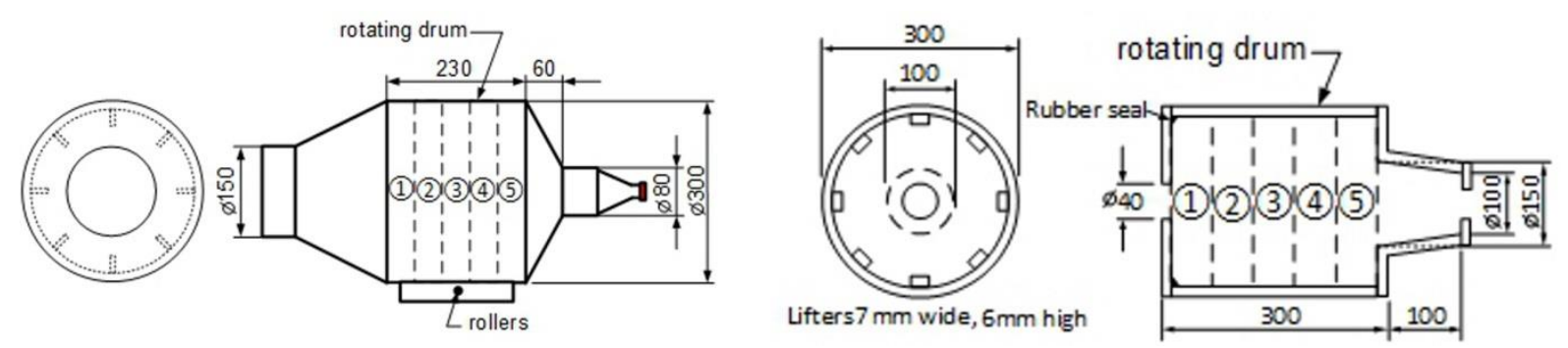

Figure 1 European Standard dustiness tester (EN 15051, 2006) (left) and Australian Standard dustiness tester (AS 4156.6, 2000) (right)

The ES tester is designed to collect the sub particles of dust from a material sample at varying moisture contents, via a series of filters in the inhalable, thoracic and respirable dust ranges. The AS tester allows the determination of the dust extinction moisture content of a material by testing samples of the same material with varying moisture contents and collecting the generated dust in a domestic vacuum cleaner bag (Frew et al., 2013).

A dustiness tester drum is similar in geometry to a horizontal drum mixer; the main difference is in their operation, with dustiness testers using a relatively small quantity of material. In comparison, the ES tester requires only $35 \mathrm{~cm}^{3}$ of material to complete a test (equivalent to $0.2 \%$ of the internal volume of the drum), whereas the AS tester states that 1000 grams of material (coal) should be used. Although the AS tester was developed for testing the dustiness of coal samples, the operation of the tester can be applied to any material which contains a dust component. With coal having an approximate loose-poured bulk density of $1000 \mathrm{~kg} / \mathrm{m}^{3}$, this equates to approximately 1 litre of material. The decision was made that when testing any other material, 1 litre of product would be used for consistency and direct comparison, equivalent to $4.7 \%$ of the internal volume of the drum. 


\subsection{Test Material}

Polyethylene pellets were used experimentally to investigate the motion of particles in both dustiness testers. The product size distribution was determined by mechanical sieving, resulting in $82 \%$ of particles in the $4.0-5.6 \mathrm{~mm}$ range, $16 \%$ in the $3.35-4.0 \mathrm{~mm}$ range and $2 \%$ in the $2.36-$ $3.35 \mathrm{~mm}$ range. In previous published work, (Wangchai et al., 2015), the lower $18 \%$ of the size distribution was discarded and a 'mono-sized' DEM investigation was completed using an averaged particle size in the $4.0-5.6 \mathrm{~mm}$ range. The larger particle size would also allow for DEM simulations to be completed quicker. For the DEM investigation presented in this paper, the effect of varying size fractions will be investigated based on an $82 \%$ / 18\% split, as explained further in Section 3.2

\subsection{Loading of Material}

For the experimental testing in the ES and AS dustiness testers, the initial positioning of the sample was varied to investigate the influence of the movement of material within the drums during rotation. Four locations were trialled in each tester; an even spread of material from front to back, a heap at the front, a heap in the middle and a heap at the back of the drum.

\section{Discrete Element Method}

DEM is a numerical technique developed by Cundell and Strack (1979) for predicting the translational and rotational motion of each particle, including collisions between each particle and between particles and boundaries (Yang et al., 2008). The DEM software used in this study (EDEM 2.5, 2013) has implemented the Hertz-Mindlin no-slip contact model (Cundall, 1988) with a springdashpot and frictional slider in the tangential direction (Tsuji Y. et al., 1992) for the particle-particle and particle-wall contacts.

\subsection{DEM Model Parameters}

For accurate DEM simulations to be produced, the particle and bulk properties of the polyethylene pellets needed to be determined, along with the interactions with the stainless steel drums and the acrylic plate at the front of the AS tester, as shown in Table 1.

Table 1 Particle and bulk properties of polyethylene pellets, stainless steel and acrylic

\begin{tabular}{lccc}
\hline Properties & Polyethylene Pellets & Stainless Steel & Acrylic \\
Particle size distribution $(4.00-5.60 \mathrm{~mm})$ & $82 \%$ & - & - \\
Particle size distribution $(3.35-4.00 \mathrm{~mm})$ & $16 \%$ & - & - \\
Particle size distribution $(2.36-3.35 \mathrm{~mm})$ & $2 \%$ & - & - \\
Particle density $\left(\mathrm{kg} / \mathrm{m}^{3}\right),\left(\rho_{s}\right)$ & 908 & 8000 & 1200 \\
Loose-poured bulk density $\left(\mathrm{kg} / \mathrm{m}^{3}\right),\left(\rho_{b}\right)$ & 532 & - & - \\
Poisson's ratio $(v)$ & 0.45 & 0.29 & 0.35 \\
Shear modulus $(\mathrm{Pa}),(G)$ & $1.17 \times 10^{8}$ & 0.65 & $1 \times 10^{9}$ \\
Particle coefficient of restitution, $(e)$ & 0.654 & 0.3 & 0.658 \\
Particle coefficient of static friction, $\left(\mu_{s}\right)$ & 0.2 & 0.1 & 0.1 \\
Particle coefficient of rolling friction, $\left(\mu_{r}\right)$ & 0.1 & & \\
\hline
\end{tabular}




\subsection{DEM Particle Shape Generation}

The particle shape used in the DEM simulations is based on the measurement of a random selection of particles (Wangchai et al., 2013). Wangchai et al. (2015) based their previous observations on the assumption the polyethylene pellets were mono-sized. This investigation attempts to provide an insight into the effect of particle size on the motion of particles within the rotating drums and also to observe any segregation that results from the variation in particle size. The base particle shape used in the previous and current DEM simulations is represented in Figure 2, with key dimensions, $\mathrm{d}_{1}=$ $3.94 \mathrm{~mm}$ and $\mathrm{d}_{2}=5.25 \mathrm{~mm}$, giving an aspect ratio $\mathrm{d}_{2} / \mathrm{d}_{1}=1.33$.

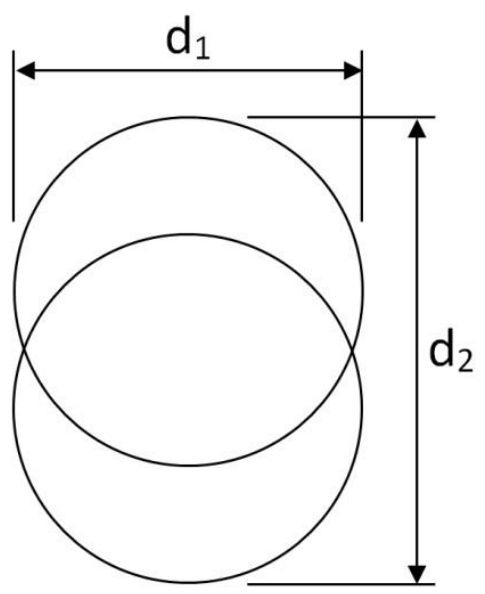

Figure 2 Representation of the polyethylene particle for the DEM simulations

Table 1 shows the measured particle size distribution of the polyethylene pellets. With only $2 \%$ of the particles being below $3.35 \mathrm{~mm}$, the decision was made to omit these particles from the simulations and instead adjust the $3.35-4.00 \mathrm{~mm}$ size range to $18 \%$ rather that $16 \%$. This results in a minor benefit in terms of simulation time, with little influence on the outcome of the simulations. In light of this, the size distribution of the polyethylene pellets was classed as binary, with $82 \%$ classed as large particles and $18 \%$ classed as small particles.

A series of simulations were devised to investigate the effect of variation in particle size, keeping the large particle, P1, the same for all simulations and varying the size of the small particle for each simulation completed. Table 2 summarises the $\mathrm{d}_{1}$ and $\mathrm{d}_{2}$ dimensions for each of the additional six small particles used in the simulations. The P2 particle is representative of the smaller sized particle measured experimentally while P3 to P7 are arbitrarily sized particles to generate increasing ratios of large to small particles. It should also be noted that for both the large and small particles used in these DEM simulations, a mono-sized particle generation was used in the setup of the simulations, based on the upper size of each size distribution. This was applied in the interests of minimising simulation time and should still provide adequate simulation results to show trends based on variation in particle size.

The calculation of the required number of large particles was based on $82 \%$ of the sample volume for both the ES and AS testers and the volume of a single large particle. Similarly, the number of small particles was based on $18 \%$ of the sample size for both the ES and AS testers and the volume of the respective small particles, also shown in Table 2. A simplification was made in the calculation of the number of particles required, that being there was no accounting for the void 
space that would exist between the particles, which has led to a slight over-prediction of the number of small and large particles required.

Table 2 Dimensions of simulated particles and the number of particles required for the DEM simulations

\begin{tabular}{|c|c|c|c|c|c|}
\hline Particle & $\begin{array}{c}\mathrm{d}_{\mathbf{1}} \\
(\mathbf{m m})\end{array}$ & $\begin{array}{c}\mathbf{d}_{2} \\
(\mathbf{m m})\end{array}$ & $\begin{array}{l}\text { Large to Small } \\
\text { Particle Size } \\
\text { Ratio }\end{array}$ & $\begin{array}{l}\text { Number of } \\
\text { Particles for } \\
\text { the ES Tester } \\
\text { Large / Small }\end{array}$ & $\begin{array}{l}\text { Number of } \\
\text { Particles for } \\
\text { the AS Tester } \\
\text { Large / Small }\end{array}$ \\
\hline P1 & 3.94 & 5.25 & - & $432 / 0$ & $12330 / 0$ \\
\hline $\mathrm{P} 2$ & 3.35 & 4.46 & $\mathrm{P} 1 / \mathrm{P} 2=1.17$ & 345 / 139 & 9825 / 3928 \\
\hline P3 & 2.63 & 3.50 & $\mathrm{P} 1 / \mathrm{P} 3=1.5$ & $345 / 295$ & $9825 / 8340$ \\
\hline $\mathrm{P} 4$ & 2.0 & 2.63 & $\mathrm{P} 1 / \mathrm{P} 4=2.0$ & $345 / 505$ & $9825 / 19700$ \\
\hline P5 & 1.57 & 2.04 & $\mathrm{P} 1 / \mathrm{P} 5=2.5$ & $345 / 1360$ & 9825 / 38420 \\
\hline P6 & 1.30 & 1.72 & $\mathrm{P} 1 / \mathrm{P} 6=3.0$ & $345 / 2350$ & 9825 / 66500 \\
\hline P7 & 1.13 & 1.48 & $\mathrm{P} 1 / \mathrm{P} 7=3.5$ & $345 / 3780$ & $9825 / 106700$ \\
\hline
\end{tabular}

\subsection{DEM Simulation Conditions}

The DEM simulations were performed using a DELL Precision T7500 workstation, with 24 GB RAM and 4 processor cores. The total simulation run time for a $60 \mathrm{sec}$ simulation varied between 60 and 120 hours, depending on the combination of particle sizes simulated. Material flow in the ES and AS rotating drums was investigated via DEM simulation using the same four different loading positions as described for the experimental tests and also for a range of different particle sizes. The simulated material heaps were randomly generated by allowing the particles to fall under gravity, with no initial velocity, from an injection plane located above the desired initial loading position in the drum, forming a natural heap. The initial homogeneous mixture of the large and small particles is achieved by the particles being randomly generated via the injection plane. After the formation of the heap was complete, the drums were rotated at $4 \mathrm{rpm}$ for the ES tester and $29 \mathrm{rpm}$ for the AS tester for a total of 60 seconds in all simulated cases.

\section{Simulation Results}

The effect of particle size ratio on the influence of particle segregation within the rotating drums was investigated from the DEM simulation outputs. By using the combination of particle sizes detailed in Table 2, the percentage of each sized particle in each of the five bins (refer Figure 1) were determined via post-processing. This analysis was performed for the entire simulation to graph the movement of polyethylene pellets throughout the rotating drums, based on the initial loading condition where the material was evenly spread along the drum, as this arrangement has shown to reach steady-state in the least amount of time (Wangchai et al, 2015).

\subsection{Axial Segregation}

For the ES tester, the percentage (volume basis) of the small size fraction at $\mathrm{t}=60 \mathrm{sec}$ within each bin has been plotted in Figure 3 for the range of particle size ratios simulated. Adding the values present in each bin for a given size ratio will yield $100 \%$, indicating the total volume of small particles present for the simulation, which in turn is $18 \%$ by volume of the total material sample added to the drum for simulation. From this information the volume fraction of the large particles in 
each bin can be inferred. Differing trends are present, however, the volume fractions of small particles in each bin fall within a relatively narrow band. This indicates that there is only minimal axial segregation occurring. The predominant reason for this is due to the small quantity of material used in each test, not allowing segregation to readily occur.

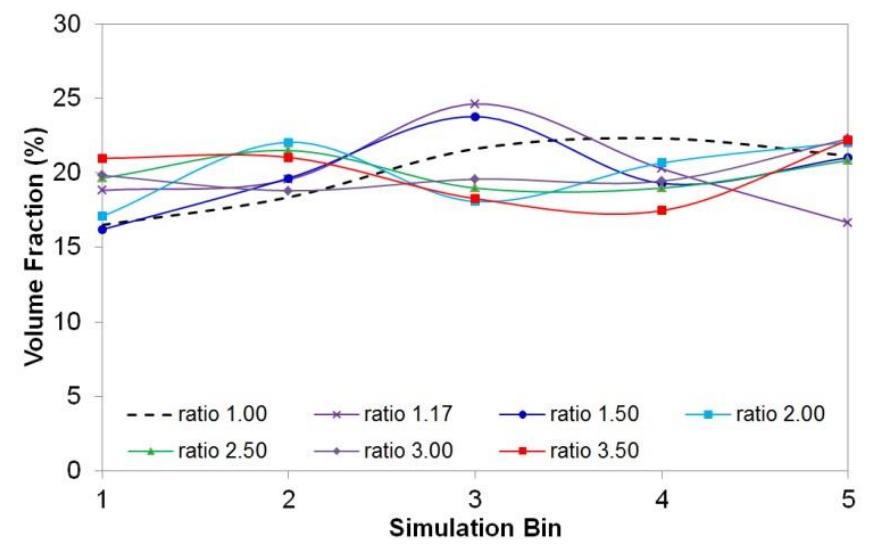

Figure 3 The volume fraction of small particles present in each bin of the ES tester for the range of particle size ratios simulated when $t=60 \mathrm{sec}$ for an initial even spread of material. Please refer to the online version of the paper for additional clarity.

An additional analysis was performed for the central bin (bin 3), where the time history (based on number of revolutions) of the percentage of small particles for the duration of a $60 \mathrm{sec}$ simulation has been extracted from the simulation data and is shown in Figure 4. Again, there is a relatively narrow band of results; however, the somewhat erratic nature of the plots can be linked to the fact that even when a small amount of material enters or leaves bin 3, this can have a substantial impact on the volume fraction, due to the small total test sample.

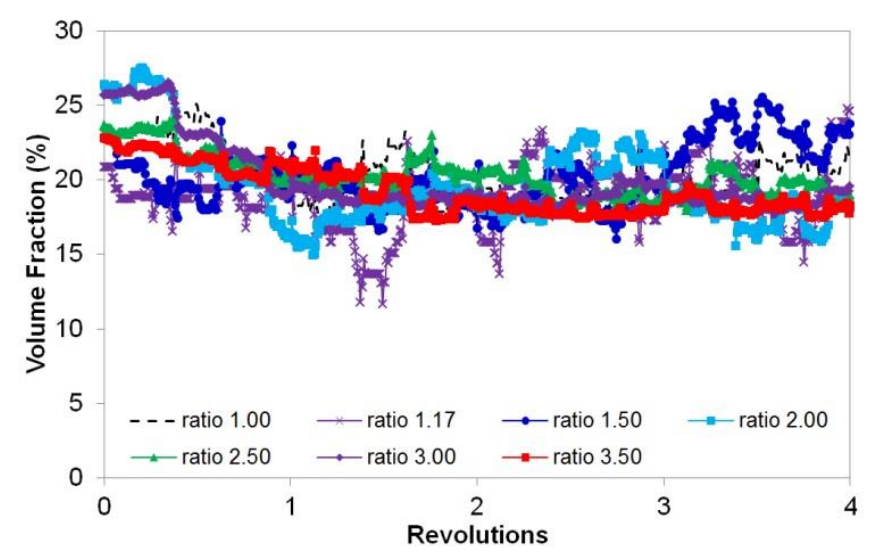

Figure 4 The trend of the small particle concentration in bin 3 of the simulated ES tester drum over a $60 \mathrm{sec}$ simulation for an initial even spread of material. Please refer to the online version of the paper for additional clarity.

A further investigation was made of all four material loading positions for bin 3 using just the size ratio of 3.5 and is presented in Figure 5. It can be seen that for the front and back loading positions, the results are very similar, which should be the case as they are mirrors of each other. The central loading position shows a rapid decrease in the fraction of small particles present as material spreads in either direction along the drum and the initial even loading of material remains constant 
throughout the test. As can be seen from Figure 5, steady-state conditions are obtained after approximately 2 revolutions $(\mathrm{t}=30 \mathrm{sec})$.

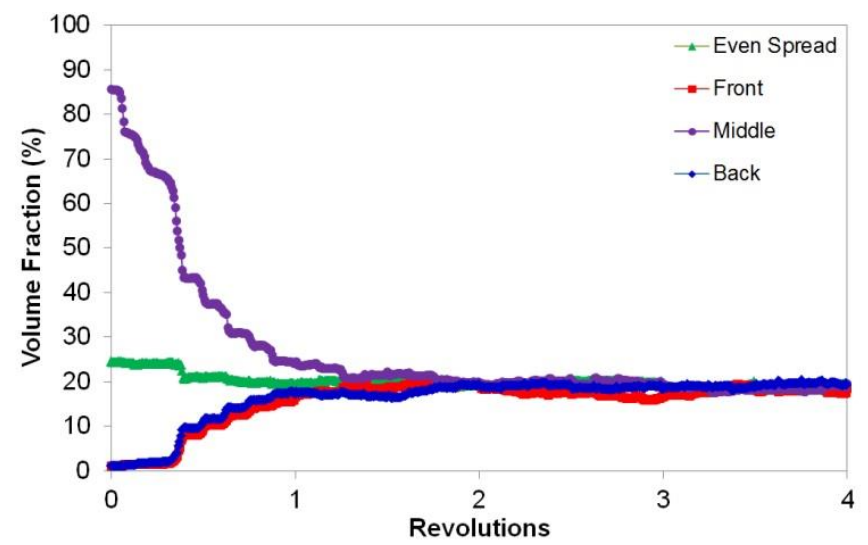

Figure 5 The volume fraction of small particles at the middle of the drum (bin 3) showing the four material loading positions in the ES tester with a size ratio of 3.5

The lack of segregation in the ES tester simulations is further evident in the set of images shown in Figure 6 for the range of particle size ratios simulated at time $t=60 \mathrm{sec}$. The small particles are shown in red (dark particles) and the large particles are shown in yellow (light particles) and it is apparent that there is a relatively even distribution of both small and large particles along the entire length of the drum in all cases, which corresponds to the plots previously shown in Figures 3 and 4.

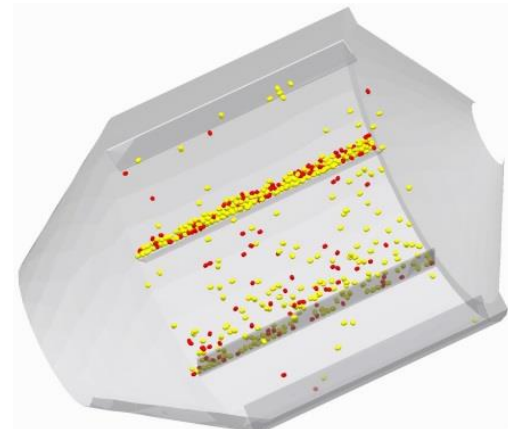

(a) ratio 1.17

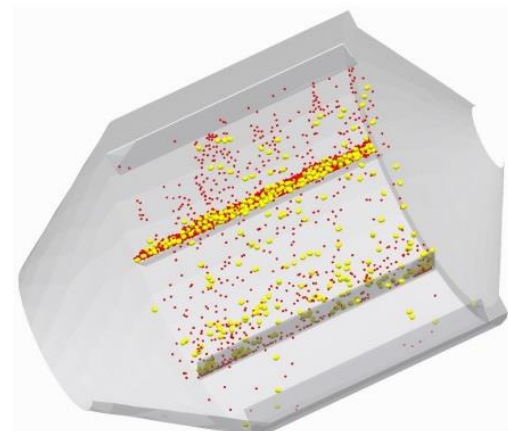

(d) ratio 2.5

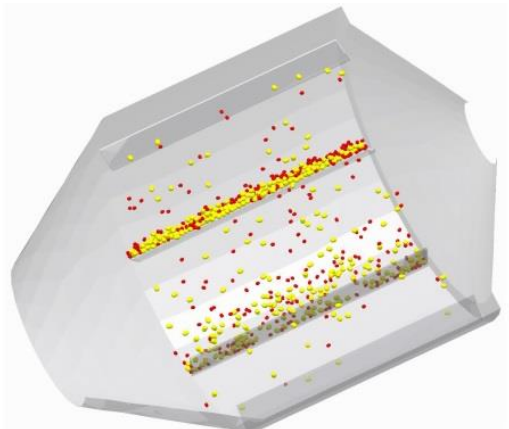

(b) ratio 1.5

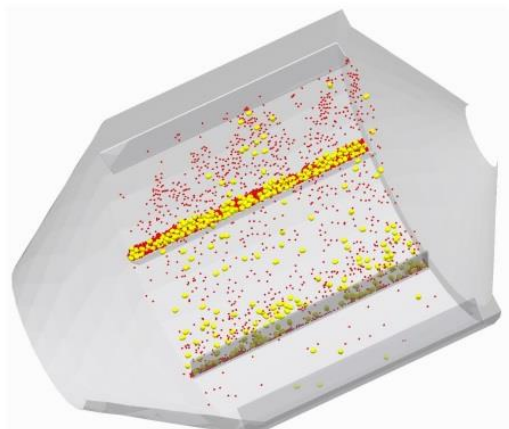

(e) ratio 3.0

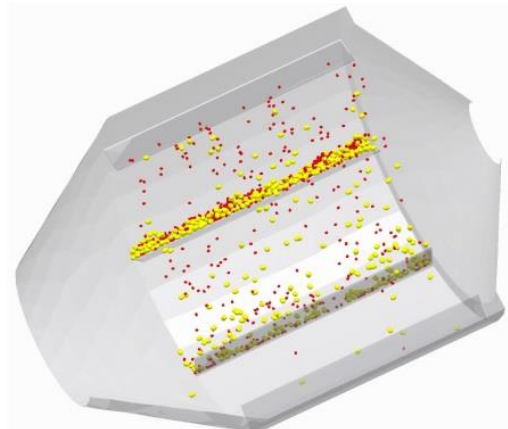

(c) ratio 2.0

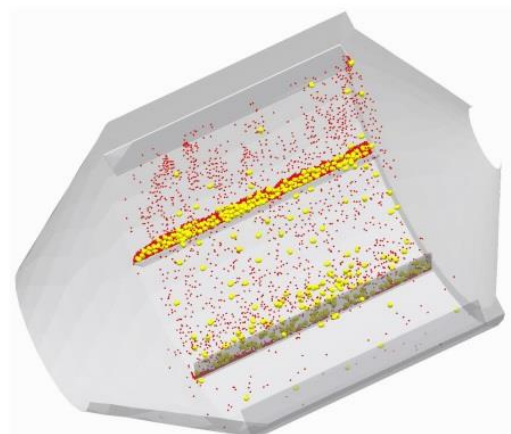

(f) ratio 3.5

Figure 6 Different particle size ratios in the ES tester at time $t=60 \mathrm{sec}$ for an initial even spread of material, showing very little axial segregation. Small particles are red (dark) and large particles are yellow (light). Please refer to the online version of the paper for additional clarity. 
For the AS tester, different results were observed for the axial segregation. The trend of the axial segregation can be seen for the range of particle size ratios tested in Figure 7, with the graph showing the percentage of small particles in each bin. When the particle size ratio is low, a larger concentration of small particles is present at both end walls of the rotating drum (bin 1 and bin 5). As seen from Table 2, there are much less small particles in the simulation than large particles for the low size ratios. As the rotating heap forms, the larger particles migrate to the top surface of the heap and then roll down the slope, over the smaller particles; which has been observed previously by Gupta (1991). The reason this is that the coefficient of friction between the particles and the end walls of the drum (stainless steel and acrylic) are higher in value than the coefficient of friction between particles, thus creating a steeper heap angle at the walls. However, the volume fraction of small particles increases at the middle of the rotating drum (bin 3) as the particle size ratio increases. This is due to the larger number of small particles in the simulations as the size ratio increases and the subsequent interaction between the small and large particles in the simulation. The result is less opportunity for the larger particles to limit to movement of the smaller sized particles, which results in higher concentration of finer particles in the centre of the drum (bin 3). The critical point where migration of fine particles reaches and moves from the equilibrium condition (evenly distributed) along the drum occurs between the size ratios of 2.0 and 2.5.

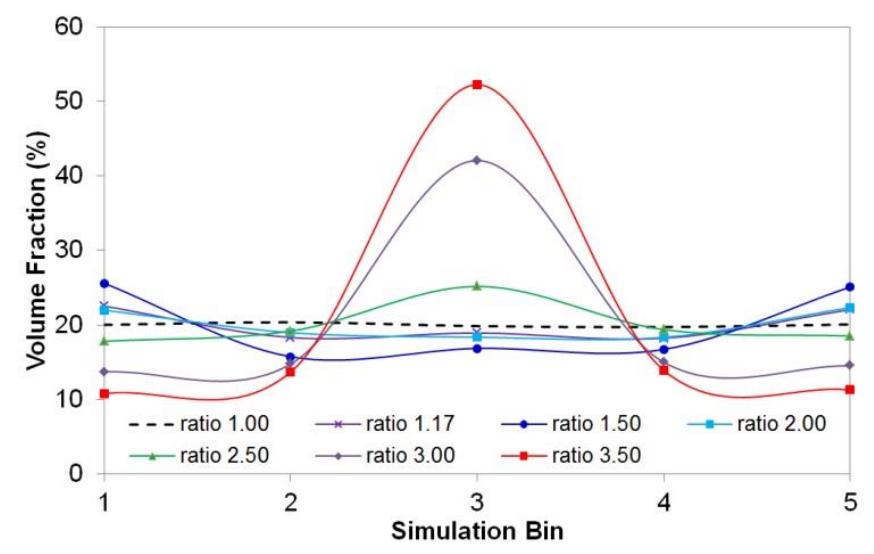

Figure 7 The volume fraction of small particles present in each bin of the AS tester for the range of particle size ratios simulated when $\mathrm{t}=60 \mathrm{sec}$ for an initial even spread of material. Please refer to the online version of the paper for additional clarity.

Isolating bin 3 for further scrutiny, the trend of the concentration of small particles has been followed over the first $60 \mathrm{sec}$ ( 29 revolutions) of a simulated test and is shown in Figure 8 covering the range of particle size ratios simulated. It can be seen that for all size ratios, a steady-state concentration of small particles has been reached after approximately 20 revolutions, equivalent to $40 \mathrm{sec}$. 


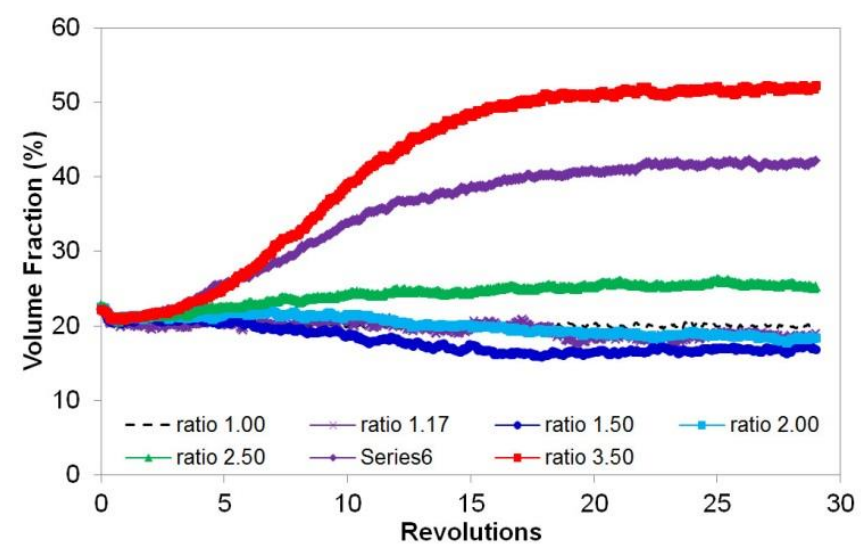

Figure 8 The trend of the small particle concentration in bin 3 of the simulated AS tester drum over a $60 \mathrm{sec}$ simulation for an initial even spread of material. Please refer to the online version of the paper for additional clarity.

A further investigation of the four material loading positions used in the AS tester simulations shows a variation in results based on particle size ratio for bin 3. Again focussing on the small particle fraction within each simulation, the time history up to $t=60 \mathrm{sec}$ has been plotted in Figure 9 for a size ratio of 1.17 and in Figure 10 for a size ratio of 3.5. These two size ratios were chosen as they cover the extremes of the size ranges used in the DEM simulations. As can be seen in Figure 9, when the size ratio is small, steady-state conditions are reached almost instantly and the results match with those shown in Figure 7 for the 1.17 size ratio. In Figure 10 it can be seen that more time is required (approx. 15 revolutions) before steady-state is achieved, where the volume fraction of small particles levels out at approximately 50\% for all loading cases. This result also matches with the previously displayed data in Figure 7 for the 3.5 size ratio and also the graphical representations shown later in Figure 11.

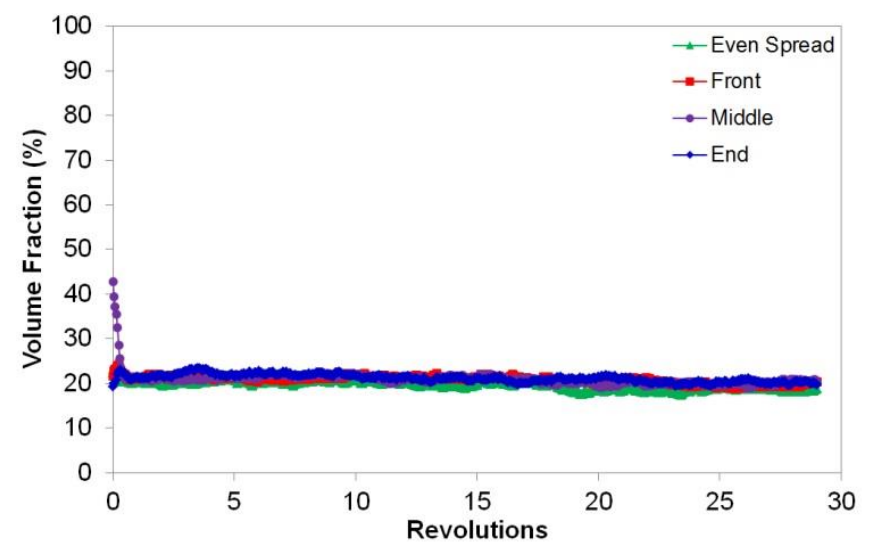

Figure 9 Shows the volume fraction at the middle of the drum with different initial heap of small particle rotate in the AS tester with a size ratio 1.17 over a $60 \mathrm{sec}$ simulation 


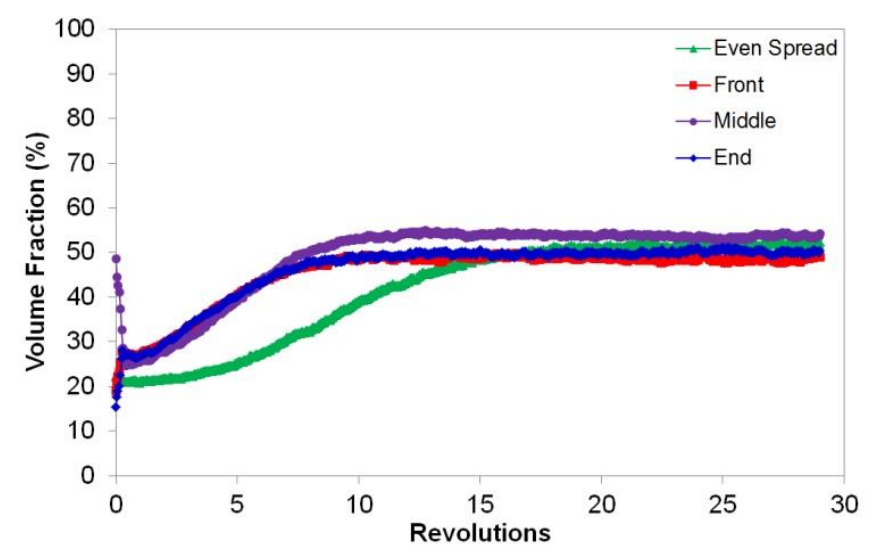

Figure 10 Shows the volume fraction at the middle of the drum with different initial heap of small particle rotate in the AS tester with a size ratio 3.50 over a $60 \mathrm{sec}$ simulation

Further examining the segregation occurring in the AS tester, Figure 11 shows the results from the range of particle size ratios simulated at time $\mathrm{t}=60 \mathrm{sec}$. There are substantially more particles present in these simulations compared to those of the ES tester and as a result, there is a clear indication that axial segregation is occurring, especially evident for size ratios of 3.0 and 3.5, with a band of small particles forming in the middle of drum (bin 3), which supports the comments previously made with respect to Figure 7. This corresponds with the noticeable jump in small particle volume fraction in bin 3 seen in Figure 7. Even though the volume of material in the drum (4.7\%) is substantially lower than for drum mixers (generally $>20 \%$ ), similar axial segregation trends are beginning to present.

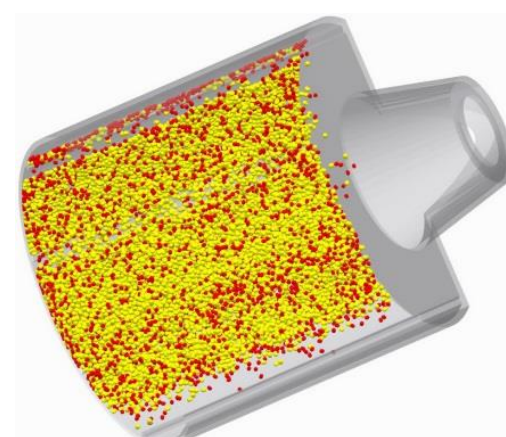

(a) ratio 1.17

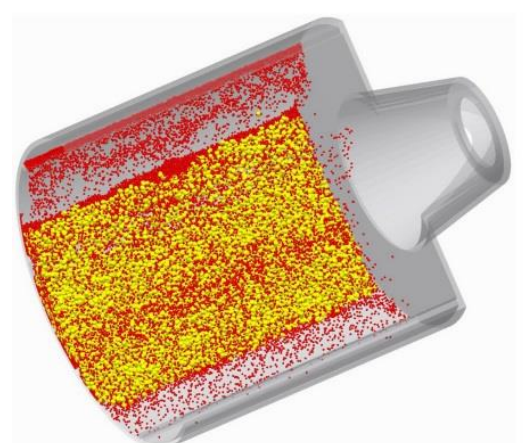

(d) ratio 2.5

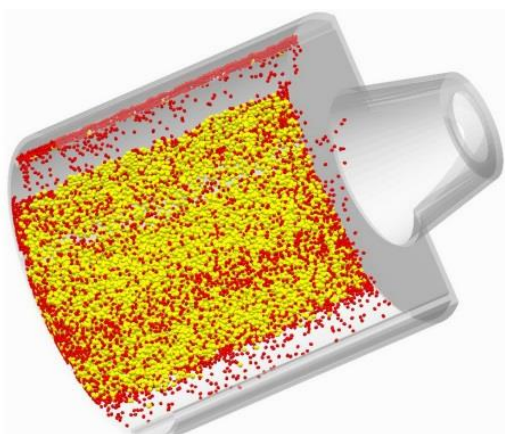

(b) ratio 1.5

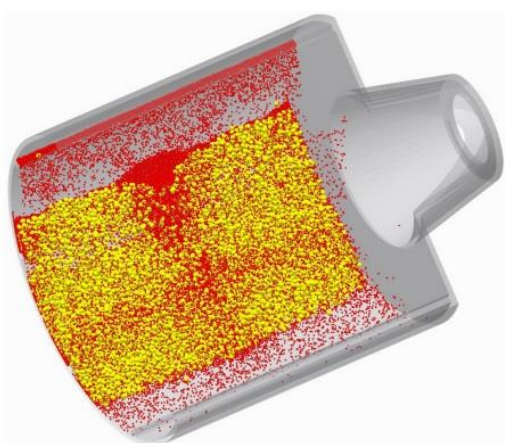

(e) ratio 3.0

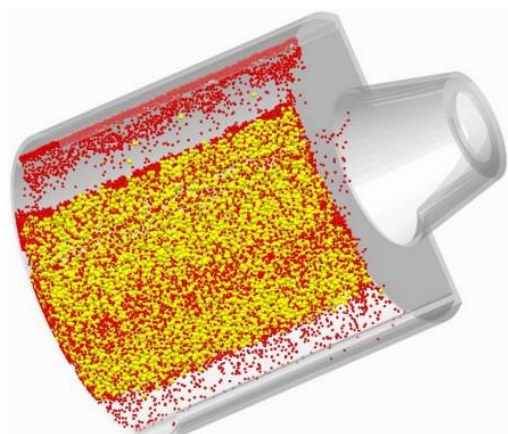

(c) ratio 2.0

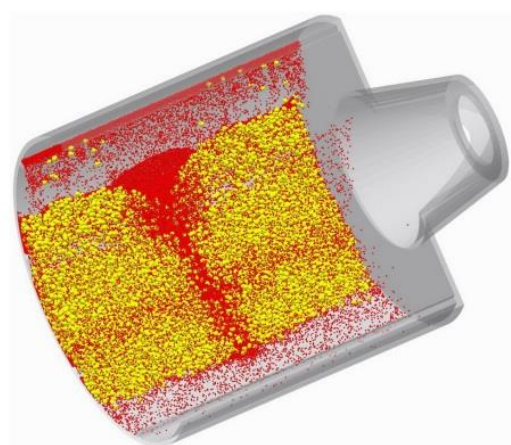

(f) ratio 3.5

Figure 11 Different particle size ratios in the AS tester at time $t=60 \mathrm{sec}$ for an initial even spread of material, showing gradual increase in axial segregation as particle size ratio increases (view from above). Small particles are red (dark) and large particles are yellow (light). Please refer to the online version of the paper for additional clarity. 


\subsection{Radial Segregation}

From the analysis of the axial segregation, the volume fraction of small particles present in each bin has already been determined. However, this data does not indicate the degree of radial segregation present within each bin. It has been well established in research focusing on drum mixers that a central core of small particles forms, surrounded by larger particles. The purpose of analysing the radial segregation is to investigate whether the same trends hold true for dustiness testers, where tests contain much smaller quantities of material. If these 'pockets' of smaller particles do exist, especially in physical testing, then there is also a possibility that they restrict the extraction of a portion of the fine dusty material for which the dustiness testers are designed to capture.

Due to the small quantity of material present for the ES dustiness tester simulations, no bed of material formed at the base of the drum, therefore no radial segregation could be observed. This is evident in Figure 12, where a cross-sectional slice of each bin has been displayed. The quantity of material being elevated by each of the lifters is not large enough to be able to distinguish any discernible segregation pattern. For the ES tester simulations, only the results for the size ratio of 3.5 with an even initial loading of material have been displayed as this ratio had the potential to show the greatest size segregation. The results for the other size ratios displayed very similar trends to those in Figure 12.

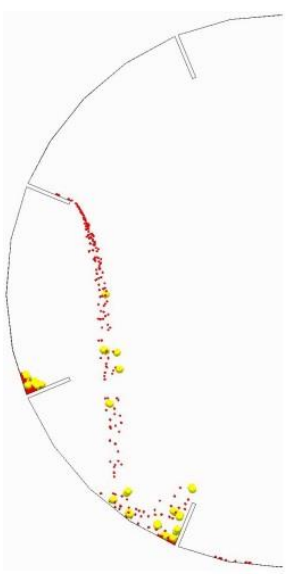

(a) Bin 1

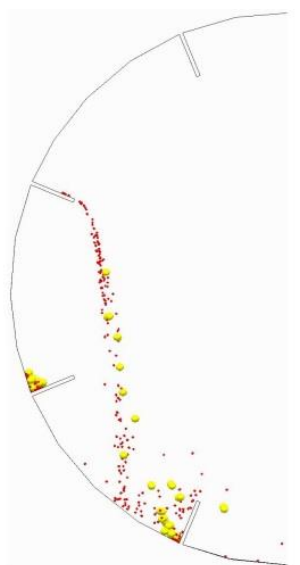

(b) Bin 2

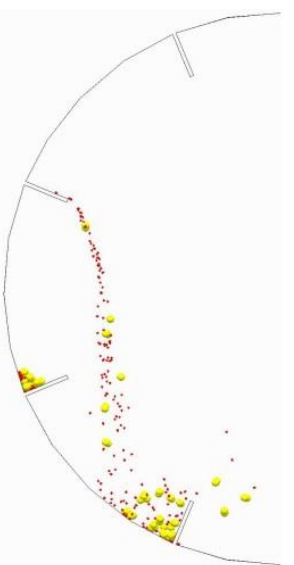

(c) Bin 3

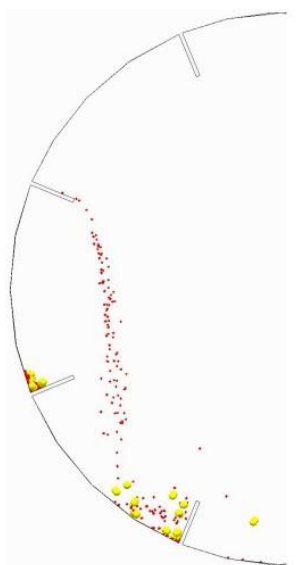

(d) Bin 4

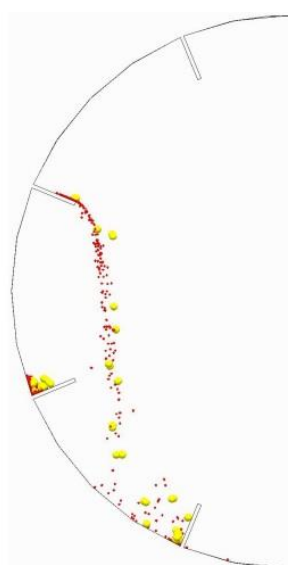

(e) Bin 5

Figure 12 Particle flow within each bin of the ES tester at $t=60 \mathrm{sec}$ for the size ratio of 3.5 and the even spread of material. Small particles are red (dark) and large particles are yellow (light). Please refer to the online version of the paper for additional clarity.

The simulations of the AS tester with the four initial loading positions using a size ratio of 3.5 are displayed in Figure 13. Slices of each bin have been produced for each material loading position to visualise any radial segregation that has occurred as a result of drum rotation. As can be seen, at time $\mathrm{t}=60 \mathrm{sec}$, there is a clear migration of small particles moving from the front and back of the drum towards the centre (bin 3). In all four loading cases, there does not seem to be a substantial difference in the segregation pattern. Another important observation is that there does not seem to be a central core of small particles forming in the large cluster of particles at the bottom of the drum. This does not match the trend seen in horizontal drum mixers, however, the relatively small volume of particles used in the dustiness tester simulations is the most likely reason for this. It is quite possible that there is a minimum critical volume of particles required to generate this central 
core of small particles, although this cannot be confirmed as this condition is outside normal operation of the dustiness testers.
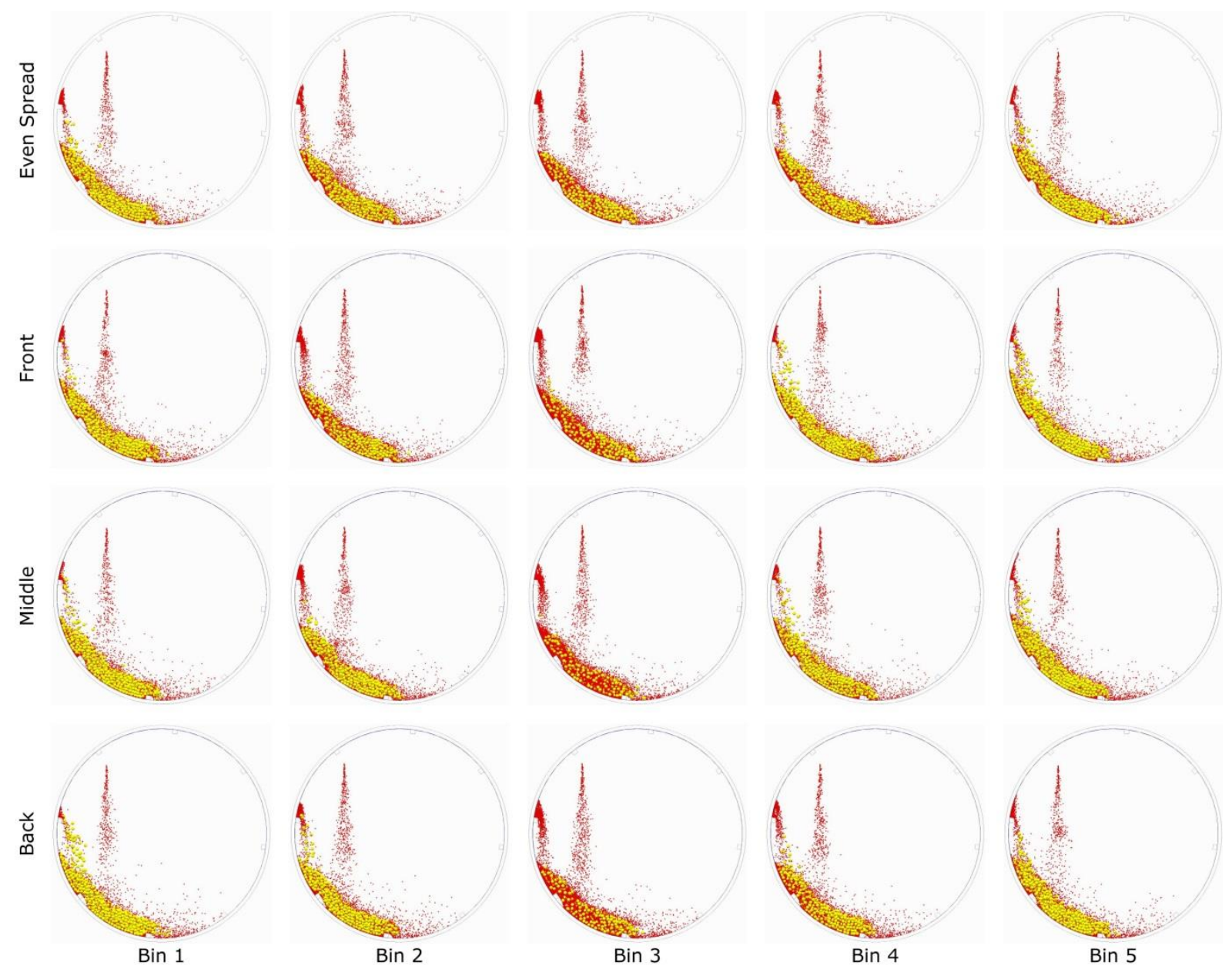

Figure 13 Particle flow within each bin of the AS tester at $t=60 \mathrm{sec}$ for the size ratio of 3.5 and four initial material locations. Small particles are red (dark) and large particles are yellow (light). Please refer to the online version of the paper for additional clarity.

\section{Conclusions}

In this study, the behaviour of different binary mixtures of size ranges of polyethylene pellets has been investigated via DEM simulations in an ES and AS dustiness tester. Analysis of the DEM simulation data for the four initial material locations showed that each had different initial transient behaviours for both dustiness testers, howeversteady-state conditions were reached in a relatively short time, as shown by the plotting of the volume fraction of the small particle component over a $60 \mathrm{sec}$ simulation. For the ES tester, it was found that regardless of the size ratio of the two polyethylene pellet components, the small quantity of product used per simulation did not allow observation or measurement of any axial or radial segregation within the rotating drum. For the AS tester, the larger quantity of product allowed the formation and observation of axial segregation along the drum length, which was of a similar style to that seen in drum mixers, containing a substantially higher volume fraction of material. For low size ratios the small particle fraction migrated towards the end walls but as the size ratio increased, the small particles migrated towards the centre of the drum, due to the effect of coefficient of friction between the particles and the walls. Unlike the trends seen by numerous researchers in drum mixers, only a single banding of smalllarge-small or large-small-large particles was observed, although this is likely due to the relatively 
short length of drum used. When considering radial segregation, there was no indication that this was occurring and is likely due to the low percentage fill of the drum (4.7\%). This fact is useful to know as the results indicate that there is no central core of small particles, which is commonly observed in rotating drum mixers. If the observations from the DEM simulations are a true indication of the mechanisms occurring in experimental equivalents, this indicates that it is highly unlikely that the extremely fine dust particles would be confined within an outer core of larger particles. This is important for the operation of dustiness testers because the dust particles are critical component of the test product in determining the dustiness of a material.

\section{Acknowledgements}

The authors would like to thank the Thailand Government and University of Wollongong for their financial support, which has allowed this research to be pursued.

\section{References}

Alexander, A., Muzzio, F. J. and Shinbrot, T. (2004). "Effects of scale and inertia on granular banding segregation". Granular Matter, Vol. 5, No. 4, pp. 171-175.

Arntz, M. M. H. D., den Otter, W. K., Beeftink, H. H., Boom, R. M. and Briels, W. J. (2013). "The influence of end walls on the segregation pattern in a horizontal rotating drum". Granular Matter, Vol. 15, No. 1, pp. 25-38.

Arratia, P. E., Duong, N. h., Muzzio, F. J., Godbole, P. and Reynolds, S. (2006). "A study of the mixing and segregation mechanisms in the Bohle Tote blender via DEM simulations". Powder Technology, Vol. 164, No. 1, pp. 50-57.

AS 4156.6 (2000). "Coal Preparation Part 6: Determination of Dust/Moisture Relationship for Coal", Standards Australia. pp. 21.

Breum, N. O. (1999). "The Rotating Drum Dustiness Tester: Variability in Dustiness in Relation to Sample Mass, Testing Time and Surface Adhesion". Annals of Occupational Hygiene, Vol. 43, No. 8, pp. 557-566.

Cantelaube, F. and Bideau, D. (1995). "Radial Segregation in a 2d Drum: An Experimental Analysis". Europhysics Letters, Vol. 30, pp. 133-138.

Chaudhuri, B., Mehrotra, A., Muzzio, F. J. and Tomassone, M. S. (2006). "Cohesive effects in powder mixing in a tumbling blender". Powder Technology, Vol. 165, No. 2, 7/13/, pp. 105-114.

Chen, P., Lochman, B. J., Ottino, J. M. and Lueptow, R. M. (2009). "Inversion of Band Patterns in Spherical Tumblers". Physical Review Letters, Vol. 102, No. 14, 04/06/, pp. 148001.

Chen, P., Ottino, J. M. and Lueptow, R. M. (2011). "Granular axial band formation in rotating tumblers: A discrete element method study". New Journal of Physics, Vol. 13.

Cleary, P. W. (2001). "Modelling Comminution Devices Using DEM". International Journal for Numerical and Analytical Methods in Geomechanics, Vol. 25, No. 1, pp. 83-105. 
Cleary, P. W., Morrison, R. and Morrell, S. (2003). "Comparison of DEM and Experiment for a Scale Model SAG Mill". International Journal of Mineral Processing, Vol. 68, No. 1-4, pp. 129 165.

Cleary, P. W. and Sinnott, M. D. (2008). "Assessing Mixing Characteristics of Particle-Mixing and Granulation Devices". Particuology, Vol. 6, No. 6, pp. 419-444.

Clement, E., Rajchenbach, J. and Duran, J. (1995). "Mixing of Granular Material in a Bidimensional Rotating Drum". Europhysics Letters, Vol. 30, No. 1, pp. 7-12.

Cui, Z., Zhao, Y., Chen, Y., Liu, X., Hua, Z., Zhou, C. and Zheng, J. (2014). "Transition of axial segregation patterns in a long rotating drum". Particuology, Vol. 13, 4//, pp. 128-133.

Cundall, P. A. (1988). "Computer simulations of dense sphere assemblies", pp. 113-123.

Cundall, P. A. and Strack, O. D. L. (1979). "A Discrete Numerical Model for Granular Assemblies". Geotechnique, Vol. 29, No. 1, pp. 47-65.

Dury, C. M. and Ristow, G. H. (1999). "Competition of mixing and segregation in rotating cylinders". Physics of Fluids, Vol. 11, No. 6, pp. 1387.

EDEM 2.5 (2013). "Theory Reference Guide, DEM Solutions, Edinburgh". pp. 19.

Elperin, T. and Vikhansky, A. (1999). "Mechanism of the onset of axial segregation in a rotating cylindrical drum filled with binary granular mixtures". Physical Review E - Statistical Physics, Plasmas, Fluids, and Related Interdisciplinary Topics, Vol. 60, No. 2 B, pp. 1946-1950.

EN 15051 (2006). "Workplace Atmospheres. Measurement of the Dustiness of Bulk Materials. Requirements and Reference Test Methods", Comité Européen de Normalisation. pp. 25.

Frew, I., Wypych, P. W. and Mar, L. (2013). "Different Modes of Dust Testing for Bulk Solids". The 11th International Conference on Bulk Materials Storage, Handling and Transportation (ICBMH 2013), The University of Newcastle, Australia, 2-4 July, Institution of Engineers, Australia, pp. 8.

Goda, T. J. and Ebert, F. (2005). "Three-Dimensional Discrete Element Simulations in Hoppers and Silos". Powder Technology, Vol. 158, No. 1-3, pp. 58-68.

Grima, A., Hastie, D., Curry, D., Wypych, P. and La Roche, R. (2011). "The Beginning of a New Era in Design: Calibrated Discrete Element Modelling". Australian Bulk Handling Review, Vol. 16, No. 6, pp. 14-21.

Gupta, S. D., Khakhar, D. and Bhatia, S. (1991). "Axial Segregation of Particles in a Horizontal Rotating Cylinder". Chemical Engineering Science, Vol. 46, No. 5, pp. 1513-1517.

Hamelmann, F. and Schmidt, E. (2005). "Methods for Dustiness Estimation of Industrial Powders". China Particuology, Vol. 3, No. 1-2, pp. 90-93.

Hastie, D. B. (2013). "Experimental Measurement of the Coefficient of Restitution of Irregular Shaped Particles Impacting on Horizontal Surfaces ". Chemical Engineering Science, Vol. 101, pp. 828-836. 
Hastie, D. B. and Wypych, P. W. (2010). "Experimental Validation of Particle Flow Through Conveyor Transfer Hoods Via Continuum and Discrete Element Methods". Mechanics of Materials, Vol. 42, No. 4, pp. 383-394.

Hilton, J. E. and Cleary, P. W. (2011). "The Influence of Particle Shape on Flow Modes in Pneumatic Conveying". Chemical Engineering Science, Vol. 66, No. 3, pp. 231-240.

Hjemsted, K. and Schneider, T. (1996). "Documentation of a Dustiness Drum Test". The Annals of Occupational Hygiene, Vol. 40, No. 6, pp. 627-643.

Ketterhagen, W. R., Curtis, J. S., Wassgren, C. R. and Hancock, B. C. (2009). "Predicting the Flow Mode from Hoppers Using the Discrete Element Method". Powder Technology, Vol. 195, No. 1, pp. $1-10$.

Kuo, H. P., Hsu, R. C. and Hsiao, Y. C. (2005). "Investigation of Axial Segregation in a Rotating Drum". Powder Technology, Vol. 153, No. 3, pp. 196-203.

Liu, X., Ge, W., Xiao, Y. and Li, J. (2008). "Granular Flow in a Rotating Drum with Gaps in the Side Wall". Powder Technology, Vol. 182, No. 2, pp. 241-249.

Muzzio, F. J., Robinson, P., Wightman, C. and Dean, B. (1997). "Sampling practices in powder blending". International Journal of Pharmaceutics, Vol. 155, No. 2, pp. 153-178.

Ottino, J. M. and Khakhar, D. V. (2000). "Mixing and Segregation of Granular Materials". Annual Review of Fluid Mechanics, Vol. 32, No. 1, pp. 55-91.

Pollard, B. L. and Henein, H. (1989). "Kinetics of radial segregation of different sized irregular particles in rotary cylinders". Canadian Metallurgical Quarterly, Vol. 28, No. 1, pp. 29-40.

Santomaso, A., Olivi, M. and Canu, P. (2004). "Mechanisms of mixing of granular materials in drum mixers under rolling regime". Chemical Engineering Science, Vol. 59, No. 16, pp. 3269-3280.

Sudah, O. S., Arratia, P. E., Alexander, A. and Muzzio, F. J. (2005). "Simulation and experiments of mixing and segregation in a tote blender". AIChE Journal, Vol. 51, No. 3, pp. 836-844.

Tsuji Y., Tanaka T. and Ishida T. (1992). "Lagrangian numerical-simulation of plug flow of cohesionless particles in a horizontal pipe". Powder Technology, Vol. 71, pp. 239-250.

Wangchai, S., Hastie, D. B. and Wypych, P. W. (2013). "The Simulation of Particle Flow Mechanisms in Dustiness Testers". The 11th International Conference on Bulk Materials Storage, Handling and Transportation (ICBMH 2013), The University of Newcastle, Australia, 2-4 July, Institution of Engineers, Australia, pp. 10.

Wangchai, S., Hastie, D. B. and Wypych, P. W. (2015). "The Investigation of Particle Flow Mechanisms of Bulk Materials in Dustiness Testers". Particulate Science and Technology, Advance online publication. http://dx.doi.org/10.1080/02726351.2015.1069430.

Wightman, C. and Muzzio, F. J. (1998a). "Mixing of granular material in a drum mixer undergoing rotational and rocking motions I. Uniform particles". Powder Technology, Vol. 98, No. 2, pp. 113124. 
Wightman, C. and Muzzio, F. J. (1998b). "Mixing of granular material in a drum mixer undergoing rotational and rocking motions II. Segregating particles". Powder Technology, Vol. 98, No. 2, pp. 125-134.

Wypych, P. W., Cook, D. M. and Cooper, P. (2005). "Controlling Dust Emissions and Explosion Hazards in Powder Handling Plants". Chemical Engineering \& Processing: Process Intensification, Vol. 44, No. 2, pp. 323-326.

Wypych, P. W. and Cooper, P. (1995). "Improving Dust-Fume Control". The 5th International Conference on Bulk Materials Storage, Handling and Transportation (ICBMH 1995), The University of Newcastle, Australia, 10-12 July 1995, Institution of Engineers, Australia, pp. 217222.

Yang, R. Y., Yu, A. B., McElroy, L. and Bao, J. (2008). "Numerical Simulation of Particle Dynamics in Different Flow Regimes in a Rotating Drum". Powder Technology, Vol. 188, No. 2, pp. $170-170$. 